\title{
Dielectric inspired scaling of polarization conversion subwavelength resonances in open ultrathin chiral structures
}

Andriy E. Serebryannikov, Mehmet Mutlu, and E. Ozbay

Citation: Appl. Phys. Lett. 107, 221907 (2015);

View online: https://doi.org/10.1063/1.4936603

View Table of Contents: http://aip.scitation.org/toc/apl/107/22

Published by the American Institute of Physics

\section{Articles you may be interested in}

Anisotropic metasurface with near-unity circular polarization conversion

Applied Physics Letters 108, 183502 (2016); 10.1063/1.4948594

A transparent $90^{\circ}$ polarization rotator by combining chirality and electromagnetic wave tunneling Applied Physics Letters 100, 051909 (2012); 10.1063/1.3682591

Polarization conversion of metasurface for the application of wide band low-profile circular polarization slot antenna

Applied Physics Letters 109, 054101 (2016); 10.1063/1.4960198

Asymmetric transmission of linearly polarized waves in terahertz chiral metamaterials Journal of Applied Physics 121, 033103 (2017); 10.1063/1.4974477

Planar metamaterial with transmission and reflection that depend on the direction of incidence Applied Physics Letters 94, 131901 (2009); 10.1063/1.3109780

Multiple-band reflective polarization converter using U-shaped metamaterial Journal of Applied Physics 115, 103505 (2014); 10.1063/1.4868076

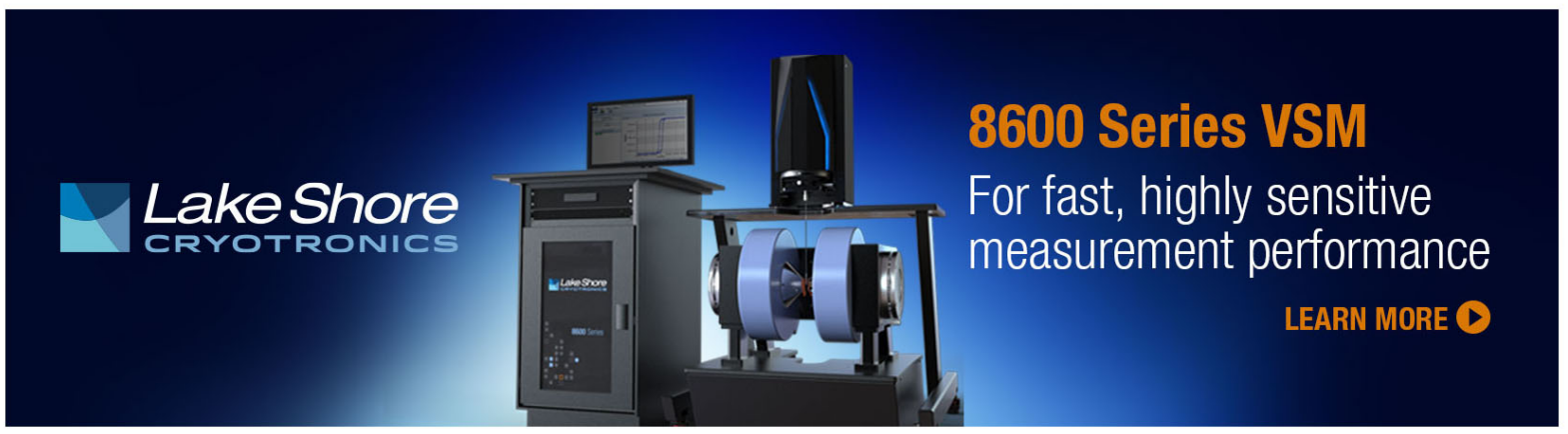




\title{
Dielectric inspired scaling of polarization conversion subwavelength resonances in open ultrathin chiral structures
}

\author{
Andriy E. Serebryannikov, ${ }^{1,2, a)}$ Mehmet Mutlu, ${ }^{2,3}$ and E. Ozbay ${ }^{2}$ \\ ${ }^{1}$ Faculty of Physics, Adam Mickiewicz University, 61-614 Poznań, Poland \\ ${ }^{2}$ Nanotechnology Research Center - NANOTAM, Bilkent University, 06800 Ankara, Turkey \\ ${ }^{3}$ Geballe Laboratory for Advanced Materials, Stanford University, Stanford, California 94305, USA
}

(Received 23 August 2015; accepted 12 November 2015; published online 1 December 2015)

\begin{abstract}
It is shown that the scaling of subwavelength resonances in open ultrathin chiral structures can be obtained by varying only the permittivity of dielectric spacers, while multiband one-way polarization conversion and related asymmetric transmission remain possible. These features are quite general and obtainable in a wide range of parameter variation. Surprisingly, the difference in the power of $\varepsilon$ for the classical $\varepsilon^{-1 / 2}$ scaling rule and the empirical rules obtained in the present letter does not exceed 22\%, giving an important entry point for future theoretical studies and design strategies. Both spectral scaling and conservation of the polarization characteristics can be achieved by using either tunneling or real-index impedance matching. The scaled structures with strong polarization and directional selectivity may have thickness of $\lambda / 100$ and smaller. () 2015 AIP Publishing LLC.

[http://dx.doi.org/10.1063/1.4936603]
\end{abstract}

Classical (full) scaling of the spectral (frequency) and field characteristics in linear lossless passive structures is based on the similarity criteria. In the lossless cavities, eigenfrequencies can be scaled by changing the permittivity $\varepsilon$ of the filling isotropic dielectric medium, according to the Maxwell equations based $\varepsilon^{-1 / 2}$-rule. In the open resonance structures, the fields can be quite strong outside the resonators, so that quantifying resonance frequencies becomes a problem. For scattering and diffraction problems, the analog of full scaling can be realized in open structures by proportionally changing all of the geometrical sizes, so that diffraction/transmission spectra are just shifted and compressed. ${ }^{1}$ At optical frequencies, losses, finiteness of plasma frequency, and nonzero kinetic energy in metallic components lead to that the scaling of resonance frequencies becomes impossible. ${ }^{1,2}$ The suitable scaling in open resonance structures due to the varying permittivity of dielectric layers at the kept sizes of all components is not guaranteed even if the losses can be neglected, as could be possible at microwave frequencies. Moreover, nothing definite can be said a priori about the secondary, e.g., field and polarization characteristics. It is known that placing a dielectric in or near subwavelength resonators can exert different effects on electromagnetic characteristics. ${ }^{3-6}$ Hence, quantifying the subwavelength resonances and understanding and predicting the electromagnetic characteristics at this dielectric inspired scaling is highly demanded for open resonance structures. However, neither proper theoretical models nor design rules are currently available.

Today, subwavelength resonators are becoming important building elements of open resonance structures for various optical and microwave applications. Bianisotropic and, in particular, ultrathin chiral structures create a big class of such structures. They have been a focus of interest in the last decade, because of having an unprecedented capability in polarization manipulation. ${ }^{7-15}$ Efforts toward dual-/

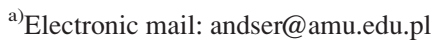

multiband and wideband one-way polarization conversion should be mentioned. ${ }^{6,11,12,15-17}$ For the chiral and bianisotropic structures, the dielectric inspired scaling of resonance frequencies is promising, but it remains unclear which scaling scenarios are realizable. The structures have recently been proposed for conversion of the linear polarization to the circular $^{10-12}$ and orthogonal linear ${ }^{11-15}$ ones and one circular polarization to the other. ${ }^{8,9}$ Perfect transmission and zero reflections for one of the two incidence directions, and zero reflections for the opposite direction are required for the most desirable regime achievable in the lossless case. ${ }^{14,18}$ The possibility of the perfect polarization conversion has been demonstrated for a general double-layer convertor. ${ }^{19}$

In this letter, we demonstrate the principal possibility of scaling and quantifying subwavelength resonances in open resonance structures, whereas the basic features of the secondary electromagnetic characteristics remain. As an example, we consider open chiral ultrathin structures, which enable efficient polarization manipulation. It will be shown that the dielectric inspired scaling of resonance frequencies can be achieved in a very wide range of variation of the dielectric substrate permittivity at constant geometrical parameters. At the same time, conservation of the ability of one-way polarization conversion does not require a special parameter adjustment and advancing the open resonance structures. The main attention is paid to polarization conversion resonances in ultrathin structures with two coupled arrays of subwavelength split-ring resonators (SRRs), which will be studied in the context of the problem of scaling. The studied scenario of scaling is partial, or unusual at least in two aspects: compared to the case when a closed resonator is fully filled with a dielectric, in our case dielectric does not fill the entire volume where resonance field might be significant and the boundaries of this volume are blurred; compared to the case when scaling is achieved by a proportional change of all geometrical parameters, our case is opposite. In order to show how general the basic mechanism is, we vary 


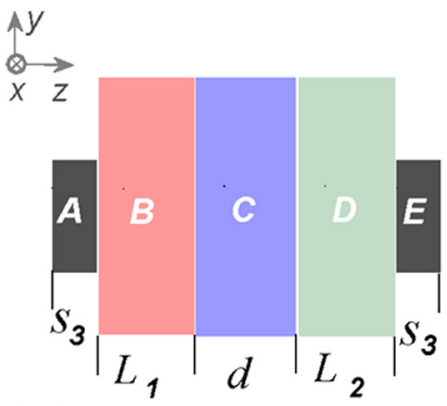

(a)

permittivity of the dielectric layers from 1 to 100 . Consideration is restricted here to microwave frequencies; the assumption of lossless metallic parts is adopted. We present here a part of our first results on scaling and quantifying subwavelength resonances, which are obtained by using CST Microwave Studio. ${ }^{20}$

Schematic of a unit cell of the studied periodic structure is shown in Fig. 1. Each SRR array and the entire structure have period of $a$ in the $x$ and $y$ directions. The width of an SRR arm is denoted by $w$. The metallic mesh with period $p<a$ consists of square holes. For the basic configuration with mesh, we take $a=22 \mathrm{~mm}, p=4.4 \mathrm{~mm}, h=2.2 \mathrm{~mm}, L_{1}=L_{2}=1.25 \mathrm{~mm}$, $d=0.5 \mathrm{~mm}, s_{1}=s_{2}=10 \mathrm{~mm}, s_{3}=1.5 \mathrm{~mm}$, and $w=3 \mathrm{~mm}$. The total thickness is $\Delta=2 s_{3}+d+L_{1}+L_{2}=6 \mathrm{~mm}$. The back-side SRR array represents the front-side SRR array rotated by $90^{\circ}$ in the clockwise direction regarding the $z$ axis. In the general case, a linearly polarized incident wave changes its polarization state when passing through such a structure. The complex amplitudes of the incident $\left(E_{x i}^{f, b}, E_{y i}^{f, b}\right)$ and transmitted $\left(E_{x}^{f, b}, E_{y}^{f, b}\right)$ waves are related by the $T$-matrix ${ }^{13,14}$

$$
\left(\begin{array}{c}
E_{x}^{f, b} \\
E_{y}^{f, b}
\end{array}\right)=\left(\begin{array}{cc}
T_{x x}^{f, b} & T_{x y}^{f, b} \\
T_{y x}^{f, b} & T_{y y}^{f, b}
\end{array}\right)\left(\begin{array}{c}
E_{x i}^{f, b} \\
E_{y i}^{f, b}
\end{array}\right),
$$

where the $x$ and $y$ components correspond to $p$ - and $s$-polarization; $T_{x x}^{f, b}$ and $T_{y y}^{f, b}$ are the co-polarized transmission coefficients; $T_{x y}^{f, b}$ and $T_{y x}^{f, b}$ are the cross-polarized transmission coefficients; and $f$ and $b$ indicate the forward (front-side illumination) and backward (back-side illumination) transmission cases. The amplitudes in these two cases are related to each other depending on the symmetries. ${ }^{13}$ At $\varepsilon=\varepsilon_{1}=\varepsilon_{2}$, we have $T_{x y}^{f}=-T_{y x}^{b}, T_{y x}^{f}=-T_{x y}^{b}$ and $T_{x x}^{f}=T_{x x}^{b}=T_{y y}^{f}=T_{y y}^{b}$.

If $T_{x x}^{f, b}=0, T_{y y}^{f, b}=0, T_{y x}^{f}=T_{x y}^{b}=0$, and $\left|T_{x y}^{f}\right|=\left|T_{y x}^{b}\right|$ $=1$, the perfect polarization conversion takes place. The scattering matrix is symmetric owing to reciprocity, so that the perfect conversion may also occur at the opposite-side illumination. In fact, transmission can be strong at either the front-side or the back-side illumination, depending on which of the two incident linear polarization states is used, and vanish at the opposite-side illumination. The use of the layers whose effect is associated with $\varepsilon<0$ and $\varepsilon>0$ in one structure may result in destructive interferences of the waves reflected at the interfaces between the layers $\mathrm{A}-\mathrm{E}$ and between air and the layers $\mathrm{A}$ and $\mathrm{E}$, enabling zero reflections and perfect tunneling. ${ }^{6,14}$ In this case, one-way (nearly) perfect transmission occurs when the specific phase conditions

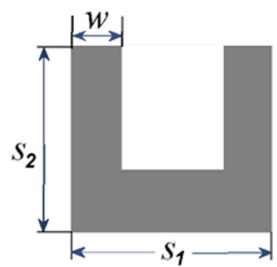

FIG. 1. (a) Schematic of a unit cell (side view): A-SRR (front side); B and $\mathrm{D}$-dielectric layers with permittivity $\varepsilon_{1}$ and $\varepsilon_{2}$, respectively; $\mathrm{C}-$ metallic mesh; E-twisted SRR (back side); (b) Metallic mesh with square holes within one structure period; (c) A twisted SRR seen from the side of layer $\mathrm{E}$. (c)

are fulfilled. On the other hand, it may be achieved in a structure without a metallic mesh, if the real-index input impedances at the interfaces are properly adjusted. ${ }^{6}$

Now, we demonstrate how subwavelength resonances enabling polarization conversion can be scaled in the basic configuration by varying $\varepsilon$ of dielectric layers $[\mathrm{B}$ and $\mathrm{D}$ in Fig. 1(a)]. To obtain the conservation of polarization characteristics, the corresponding matching regimes must be kept. Figure 2 presents the results for the basic configuration with
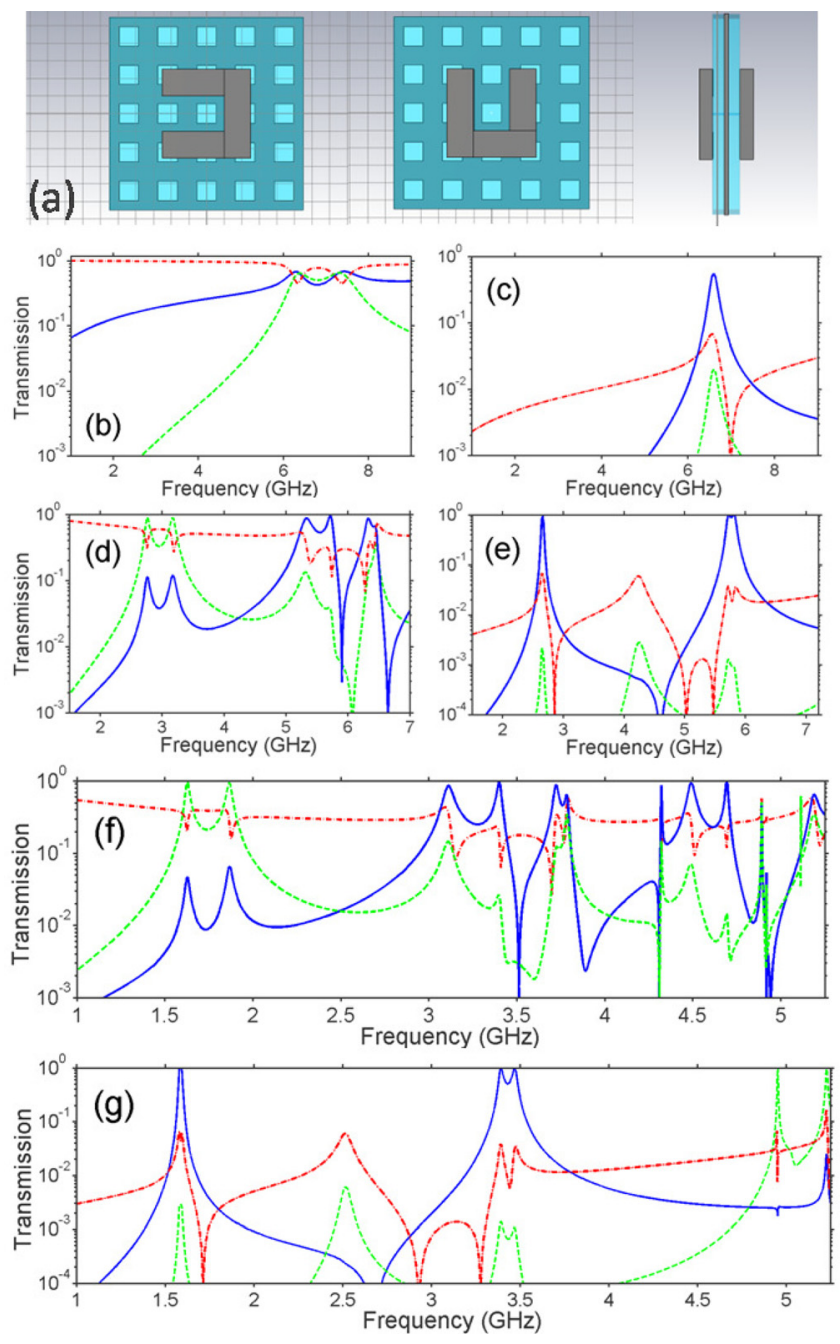

FIG. 2. (a) Front, back, and side view of a unit cell with mesh and transmission for the basic configuration (b), (d), and (f) without mesh and (c), (e), and (g) with mesh; (b) and (c) $\varepsilon=1$, (d) and (e) $\varepsilon=11.4$, (f) and (g) $\varepsilon=35.4$; solid blue line- $\left|T_{x y}^{f}\right|=\left|T_{y x}^{b}\right|$, dashed green line- $\left|T_{y x}^{f}\right|=\left|T_{x y}^{b}\right|$, and dashed-dotted red line- $-T_{x x}^{f}=T_{x x}^{b}=T_{y y}^{f}=T_{y y}^{b}$; dielectric layers in the front and back view in plot (a) are transparent. 
and without mesh. In the latter case, i.e., in Figs. 2(b), 2(d), and 2(f), thickness of the single dielectric layer between the SRR arrays is $L=L_{1}+L_{2}=3 \mathrm{~mm}$. Strong but imperfect conversion of the incident linear polarization is observed in Figs. 2(b) and 2(c) between $6 \mathrm{GHz}$ and $8 \mathrm{GHz}$. In Fig. 2(d), five bands of the nearly perfect one-way polarization conversion and related asymmetric transmission are obtained at $a / \lambda<0.5(f<6.8 \mathrm{GHz})$ due to the two maxima of $T_{y x}^{f}$ $\left(T_{y x}^{b} \approx 0\right)$ and three maxima of $T_{x y}^{f}\left(T_{x y}^{b} \approx 0\right)$. In Fig. 2(e), two one-way polarization conversion bands occur at $f=2.66 \mathrm{GHz}\left(\max \left|T_{x y}^{f}\right|=0.94\right)$ and at $f=5.76 \mathrm{GHz}\left(\left|T_{x y}^{f}\right|\right.$ $>0.995$ ), where the twin maximum creates a broad band due to the resonances overlapping. The twin maxima in Figs. 2(b), 2(d), and 2(e) are typical for twisted SRR arrays; strength of coupling can be quantified using the Lagrange formalism. ${ }^{21}$

We consider now a high- $\varepsilon$ material with $\varepsilon=35.4$. The comparison of Fig. 2(d) with Fig. 2(f) and Fig. 2(e) with Fig. $2(\mathrm{~g})$ indicates the signatures of scaling. Indeed, the dependencies of the $T$-matrix components at $\varepsilon=11.4$ and $\varepsilon=35.4$ look similar but shifted in the latter case toward lower frequencies while the secondary characteristics related to multiband one-way polarization conversion are kept. In Fig. 2(f), more than five one-way bands with either $\left|T_{y x}^{f}\right|>0.9$ $\left(T_{y x}^{b} \approx 0\right)$ or $\left|T_{x y}^{f}\right|>0.9\left(T_{x y}^{b} \approx 0\right)$ occur at $\Delta / \lambda<0.11$ and $a / \lambda<0.4(f<5.3 \mathrm{GHz})$ due to the real-index impedance matching. Similar one-way bands are also observed for the structure with the mesh in Fig. $2(\mathrm{~g})$. Here, several bands with either $\left|T_{x y}^{f}\right|>0.99\left(T_{x y}^{b} \approx 0\right)$ or $\left|T_{y x}^{f}\right|>0.99\left(T_{y x}^{b} \approx 0\right)$ appear at $a / \lambda<0.4$. Note a peak twinning that occurs (but not well seen) in Fig. 2(g) at $f \approx 1.59$ GHz. In Figs. 2(e) and 2(g), we have $Q<10^{2}$ for the lowest transmission maxima. The possible functions of the dielectric layers include downshifting the polarization conversion resonances and improving phase and/or real-index impedance matching. The SRR arrays enable polarization conversion and contribute to the matching mechanism. The mesh suppresses the unwanted $T$-matrix components and together with the other layers contributes to the tunneling related transmission.

Next, simulations were performed for numerous structures which differ from those in Fig. 2 just in the $\varepsilon$-value, for evidence of scaling. The results are presented in Table I. They confirm that the dielectric inspired scaling occurs in the basic configuration with the mesh, i.e., the resonance frequency varies monotonously with increase of $\varepsilon$. As desired, it is accompanied with strong polarization conversion. Here,

TABLE I. Comparison of the lowest resonances of $\left|T_{x y}^{f}\right|$ for various dielectric layers in the basic configuration with mesh.

\begin{tabular}{lcccccc}
\hline \hline$\varepsilon$ & $\sqrt{\varepsilon}$ & $\mathrm{f}(\mathrm{GHz})$ & $\sqrt{\alpha}$ & $\Delta / \lambda$ & $a / \lambda$ & $\left|T_{x y}^{f}\right|$ \\
\hline 1.0 & 1.0 & 6.59 & 1.0 & 0.132 & 0.483 & 0.54 \\
2.1 & 1.45 & 5.29 & 1.25 & 0.106 & 0.388 & 0.655 \\
11.4 & 3.38 & 2.66 & 2.48 & 0.053 & 0.195 & 0.935 \\
19.4 & 4.41 & 2.085 & 3.16 & 0.042 & 0.153 & 0.99 \\
27.4 & 5.24 & 1.787 & 3.69 & 0.036 & 0.131 & $>0.99$ \\
35.4 & 5.95 & 1.587 & 4.18 & 0.031 & 0.116 & $>0.99$ \\
\hline \hline
\end{tabular}

$\sqrt{\alpha}=f^{(2)} / f^{(1)}$, where $f^{(2)}$ and $f^{(1)}$ are the lowest resonance frequencies at $\varepsilon=\varepsilon^{(2)}=1$ and $\varepsilon=\varepsilon^{(1)}>1 . \sqrt{\varepsilon}-\sqrt{\alpha}$ is not very large, i.e., scaling appears in line with the rule that is similar to the classical rule, $f(\varepsilon)=A \varepsilon^{-1 / 2}$ ( $A$ is a constant). Scaling for the other resonances in the structures with the mesh and for all resonances in the mesh-free structures occurs in the same manner. For instance, for the lower resonance of the second pair, $\sqrt{\alpha^{(h)} / \alpha^{(l)}} \approx 1.7$ in Figs. 2(d) and 2(f), and $\sqrt{\alpha^{(h)} / \alpha^{(l)}} \approx 1.69$ in Figs. 2(e) and 2(g); $(h)$ and $(l)$ correspond to $\varepsilon=\varepsilon^{(h)}=35.4$ and $\varepsilon=\varepsilon^{(l)}=11.4$; $\sqrt{\varepsilon^{(h)} / \varepsilon^{(l)}} \approx 1.76$. For the lower resonance peak of the first pair in Figs. 2(d) and 2(f), we obtain $\sqrt{\alpha^{(h)} / \alpha^{(l)}} \approx 1.7$, while $\sqrt{\alpha^{(h)} / \alpha^{(l)}} \approx 1.68$ for such a peak in Figs. 2(e) and 2(g), see Table I. For the data in Table I, $\sqrt{\alpha}$ can approximately be presented as

$$
\sqrt{\alpha}=\varepsilon^{\beta}
$$

with $\beta=0.39$, while $\beta=1 / 2$ for the classical rule. Generally speaking, the fact that $\beta<1 / 2$ can be connected with that dielectric does not occupy the entire volume, in which the resonance field is significant. It is expected that the values of $\beta<1 / 2$ can also be obtained in closed resonators that are partially filled with a dielectric. The obtained results indicate that the impedance matching conditions in the mesh-free structures and the tunneling relevant phase conditions in the structures with the mesh are kept at the maxima within a wide $\varepsilon$-range, while scaling of resonance frequencies is realized together with high-efficiency one-way polarization conversion. If the phase conditions are not fulfilled, the latter is impossible in a structure with an evanescent-wave mesh. Note that the signatures indicating the possibility of similar scaling as in Table I have recently been found in the structures based on the coupled aperture (complementary SRR) arrays. ${ }^{6}$ However, the results presented in Ref. 6 are insufficient to quantify the subwavelength resonances, since only two dielectric materials have been compared therein.

Now, we demonstrate that the above discussed features of scaling and polarization conversion are general and can be obtained at smaller $\Delta$. The results are presented in Fig. 3 for the thin configuration, for which we take $L_{1}=L_{2}$ $=0.25 \mathrm{~mm}, s_{3}=0.5 \mathrm{~mm}$ and same remaining parameters as for the basic configuration, i.e., $\Delta=2 \mathrm{~mm}$. The peak widths correspond here to $Q<10^{3}$, whereas $Q>10^{3}$ can be achieved at least when $\varepsilon>35.4$. $\left|T_{x y}^{f}\right|>0.98$ for all of the one-way polarization conversion resonances and $\left|T_{x y}^{f}\right|>$ 0.99 for most of them, regardless of the value of $\varepsilon$. Recently, the very thin configuration has been studied, for which $L_{1}=$ $L_{2}=d=0.25 \mathrm{~mm}, s_{3}=5 \mu \mathrm{m}$, and $\Delta=0.76 \mathrm{~mm}$. The lowest twin maximum of $\left|T_{x y}^{f}\right| \approx 1$ again remains in a wide range of $\varepsilon$ variation. The selected results for the both thin configurations are presented in Table II. Accordingly, Eq. (2) with $\beta=0.44$ and $\beta=0.45$ can be used, at least if $\varepsilon<40$. Hence, both $1 / 2-\beta$ and $\sqrt{\varepsilon}-\sqrt{\alpha}$ are decreased and, thus, the rule of $\varepsilon^{-1 / 2}$ is approached. Note that better fitting can be achieved while a narrower range of $\varepsilon$-variation is considered. A smaller value of $\beta$ is required, for instance, if $3<\varepsilon<12$. 


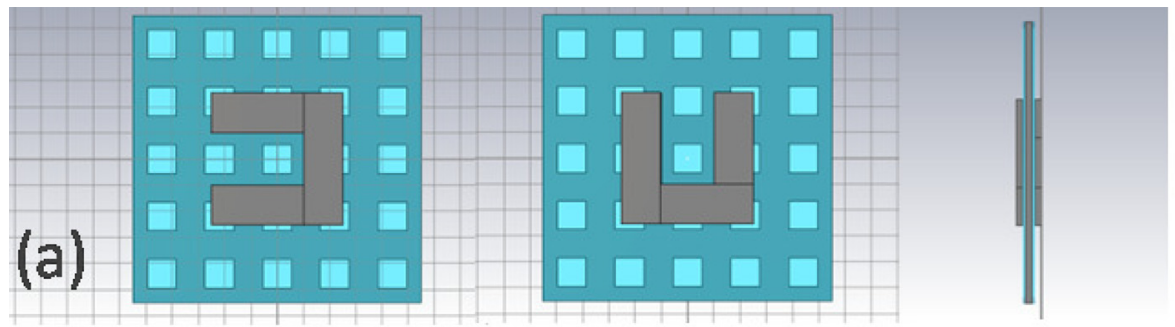

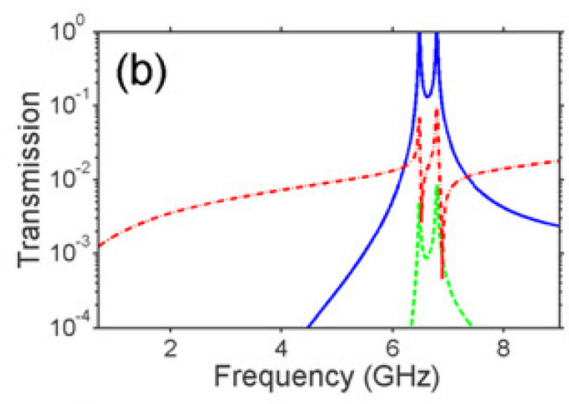

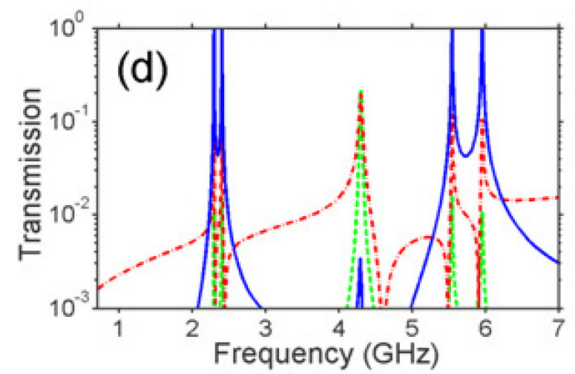

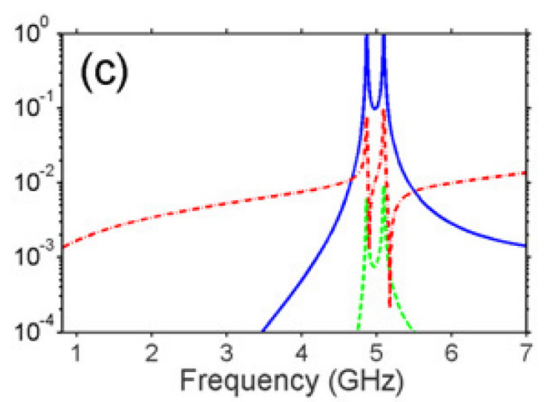

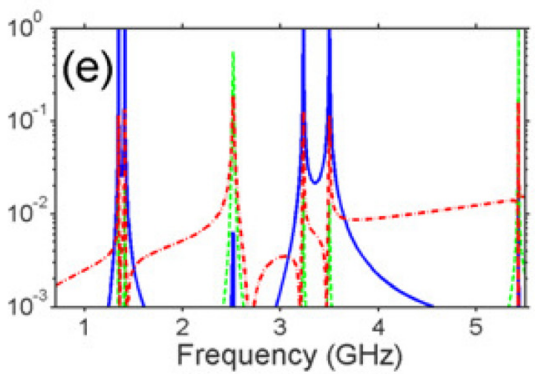

FIG. 3. (a) Front, back, and side view of the unit cell and transmission for the thin configuration with mesh at (b) $\varepsilon=1$, (c) $\varepsilon=2.1$, (d) $\varepsilon=11.4$, and (e) $\varepsilon=35.4$; solid blue line- $\left|T_{x y}^{f}\right|=\left|T_{y x}^{b}\right|$, dashed green line- $\left|T_{y x}^{f}\right|=\left|T_{x y}^{b}\right|$, and dasheddotted red line $-T_{x x}^{f}=T_{x x}^{b}=T_{y y}^{f}=T_{y y}^{b}$; dielectric layers in front and back view in plot (a) are transparent.
To summarize, a simple, dielectric inspired scaling can be obtained in the complex open resonance structures with fixed geometrical parameters, whereas multiband one-way polarization conversion and related asymmetry in transmission are conserved. The realized scaling ratios of resonance frequencies are quite close to the classical scaling rule of $\varepsilon^{-1 / 2}$. Up to five bands of perfect one-way conversion can be obtained at total thickness $\Delta<\lambda / 40$ for the dielectric layers with $\varepsilon=35.4$, while the lowest resonance can correspond to $\Delta<\lambda / 300$. These features can be obtained with and without tunneling, at various thicknesses of the structural components. The obtained results give an example of quantifying subwavelength resonances that can be useful for a much wider class of the open resonance structures than those studied in this letter, providing a proper entry point for understanding and ability of prediction of important features in the behavior of their transmission and polarization characteristics. The general concept is expected to be applicable in a

TABLE II. Comparison of the lowest resonances of $\left|T_{x y}^{f}\right|$ in thin and very thin configurations with mesh.

\begin{tabular}{lccccccc}
\hline \hline$\varepsilon$ & $\sqrt{\varepsilon}$ & $\mathrm{f}(\mathrm{GHz})$ & $\begin{array}{c}\sqrt{\alpha} \\
\text { Thin }\end{array}$ & $\Delta / \lambda$ & $\mathrm{f}(\mathrm{GHz})$ & $\begin{array}{c}\sqrt{\alpha} \\
\text { Very thin }\end{array}$ & $10^{2} \times \Delta / \lambda$ \\
\hline 1.0 & 1.0 & 6.485 & 1.0 & 0.043 & 6.367 & 1.0 & 1.61 \\
2.1 & 1.45 & 4.872 & 1.33 & 0.032 & 4.705 & 1.35 & 1.19 \\
11.4 & 3.38 & 2.297 & 2.82 & 0.015 & 2.184 & 2.92 & 0.553 \\
19.4 & 4.41 & 1.774 & 3.66 & 0.0116 & 1.686 & 3.78 & 0.427 \\
27.4 & 5.24 & 1.507 & 4.30 & 0.010 & 1.418 & 4.49 & 0.359 \\
35.4 & 5.95 & 1.347 & 4.81 & 0.009 & 1.245 & 5.11 & 0.315 \\
100 & 10 & 0.804 & 8.07 & 0.005 & 0.766 & 8.42 & 0.192 \\
\hline \hline
\end{tabular}

wide frequency range, e.g., from acoustic to terahertz frequencies. A systematic study of scaling in other types of open resonance structures, both enabling and not enabling polarization conversion, and building a general theoretical framework will be the subjects of further studies.

This work was supported by the projects DPT-HAMIT, ESF-EPIGRAT, and NATO-SET-181, and by TUBITAK under the Project Nos. 107A004, 109A015, and 109E301. A.E.S. thanks TUBITAK for partial support in the framework of the Visiting Researcher Program and National Science Center of Poland for financial assistance (Project MagnoWa DEC-2-12/07/E/ST3/00538). E.O. acknowledges partial support from the Turkish Academy of Sciences.

${ }^{1}$ T. Zentgraf, T. P. Meyrath, A. Seidel, S. Kaiser, H. Giessen, C. Rockstuhl, and F. Lederer, Phys. Rev. B 76, 033407 (2007).

${ }^{2}$ M. W. Klein, C. Enkrich, M. Wegener, C. M. Soukoulis, and S. Linden, Opt. Lett. 31, 1259 (2006).

${ }^{3}$ Y. Yao, M. A. Kats, P. Shankar, Y. Song, J. Kong, M. Loncar, and F. Capasso, Nano Lett. 14, 214 (2014).

${ }^{4}$ K. Song, X. Zhao, Y. Li, Q. Fu, and C. Luo, Appl. Phys. Lett. 103, 101908 (2013).

${ }^{5}$ X. Xia, Y. Sun, H. Yang, H. Feng, L. Wang, and C. Gu, J. Appl. Phys. 104, 033505 (2008).

${ }^{6}$ A. E. Serebryannikov, M. Beruete, M. Mutlu, and E. Ozbay, Opt. Express 23, 13517 (2015).

${ }^{7}$ C. E. Kriegler, M. S. Rill, S. Linden, and M. Wegener, IEEE J. Sel. Top. Quantum Electron. 16, 367 (2010).

${ }^{8}$ C. Pfeiffer, C. Zhang, V. Ray, L. J. Guo, and A. Grbic, Phys. Rev. Lett. 113, 023902 (2014).

${ }^{9}$ R. Singh, E. Plum, C. Menzel, C. Rockstuhl, A. K. Azad, R. A. Cheville, F. Lederer, W. Zhang, and N. I. Zheludev, Phys. Rev. B 80, 153104 (2009).

${ }^{10}$ M. Mutlu, A. E. Akosman, A. E. Serebryannikov, and E. Ozbay, Opt. Lett. 36, 1653 (2011). 
${ }^{11}$ X. Ma, C. Huang, M. Pu, C. Hu, Q. Feng, and X. Luo, Opt. Express 20, 16050 (2012).

${ }^{12}$ H.-X. Xu, G.-M. Wang, M. Q. Qi, T. Cai, and T. J. Cui, Opt. Express 21, $24912(2013)$

${ }^{13}$ C. Menzel, C. Helgert, C. Rockstuhl, E.-B. Kley, A. Tuennermann, T. Pertsch, and F. Lederer, Phys. Rev. Lett. 104, 253902 (2010).

${ }^{14}$ M. Mutlu, A. E. Akosman, A. E. Serebryannikov, and E. Ozbay, Phys. Rev. Lett. 108, 213905 (2012).

${ }^{15}$ J. Shi, X. Liu, S. Yu, T. Vu, Z. Zhu, H. F. Ma, and T. J. Cui, Appl. Phys. Lett. 102, 191905 (2013).
${ }^{16}$ Y. Xu, Q. Shi, Z. Zhu, and J. Shi, Opt. Express 22, 25679 (2014).

${ }^{17}$ J. H. Shi, H. F. Ma, C. Y. Guan, Z. P. Wang, and T. J. Cui, Phys. Rev. B 89, 165128 (2014).

${ }^{18}$ A. E. Serebryannikov, A. O. Cakmak, and E. Ozbay, Opt. Express 20, 14980 (2012).

${ }^{19}$ D. L. Markovich, A. Andriyieuski, M. Zalkovskij, R. Malureanu, and A. V. Lavrinenko, Appl. Phys. B 112, 143 (2013).

${ }^{20}$ See www.cst.com for software description.

${ }^{21}$ N. Liu, H. Liu, S. Zhu, and H. Giessen, Nat. Photonics 3, 157 (2009). 